\title{
EDUCAR PERSONAS SOLIDARIAS. EL LABERINTO DE LA EDUCACIÓN EN VALORES
}

\author{
José Ángel Paniego \\ INTERMON - Madrid
}

RESUMEN. La Educación en Valores es una realidad sobre la que existen muchas propuestas pero que suelen ser bastante deficientes. Por ello, es algo mal valorado por muchos docentes y visto como una pérdida de tiempo. Este artículo intenta mostrar qué elementos tienen las propuestas que si han funcionado, desarrollando actitudes y conductas solidarias y mejorando globalmente la calidad de la enseñanza. Estas propuestas incluyen:

- Unos valores cooperativos que sean válidos fuera de la escuela, para la vida.

- Una mentalidad del profesorado como formador global y no como impartidor de conocimientos de una materia.

- Una revisión global del quehacer educativo que incluya métodos pedagógicos y organizacionales coherentes y un análisis de valores transmitidos por el curriculum oculto.

- Un modelo de creación personal de valores por el alumnado (basado en vivenciación/problematización, análisis y actuación) que evite el adoctrinamiento y enseñe a pensar autónomamente.

- Un trabajo equilibrado de todos los niveles: de las realidades sociales (trabajadas de forma interrelacional), de los medios de socialización, de las habilidades instrumentales, de la dinámica del grupo clase y del desarrollo personal.

El uso de este modelo permite que la Educación en Valores sea un elemento que mejore la calidad de la enseñanza, prepare a la persona para su vida futura y dote a la sociedad de las personas que necesita para ser una sociedad dinámica y de progreso.

ABSTRACT. Education for values is a reality; althught lost of proposals exist, these are usually very deficient. That's why this hasn't been valued by meny teacher and seen as a waste of time. This article is going to show what elements have the proposals that have worked out developing solidarity actitudes and behaviors, and improving in general the quality of teaching. This proposals include:

- Cooperative values valid out of school; for life.

- A teacher's mentality as a global educator and not only as a teacher teaching a subject.

- A global revision of all the educational practice, including coherent pedagogical methos and a school organization, and analiced values transmited throught the hidden curriculum.

- A way in which the students can create their values, based on feelin-troubling, analyzing and doing, avording indoctrination and showing people how to think by themselves.

- A balance development of all the different levels: Social realitis (carried out all at the same time), socialization resources, instrumental habilities, dynamic of the class group and personal development.

The use of this model allows that education for values is an element that improves the quality of teaching, prepares the person for her/his future life and gives the society the people that it's need to becam an dynamic and progressive society. 
Educar a las personas en valores es hoy en día un tópico común. Especialmente tras la promulgación de la L.O.G.S.E. hemos vivido un aluvión de publicaciones, programas y propuestas de todo tipo, muchas incluso antagónicas. Muchísimos docentes, se encuentran entre un discurso de intenciones por parte de la administración sin que se aporten recursos concretos; y un derroche de materiales desde diversas asociaciones que no dejan de ser parciales y difícilmente integrables en sus clases. Si a ello le unimos la presión hacia los contenidos y formas docentes clásicas, es lógico que muchos caigan en la confusión y el desánimo. Este artículo aporta una propuesta que recoge los elementos que sí han funcionado desarrollando actitudes y conductas solidarias y mejorando globalmente la calidad de la enseñanza.'

Empecemos de una forma no demasiado ortodoxa. A continuación hay una serie de frases oídas a docentes diversos, pedimos que el lector las analice y juzgue la parte de verdad o falsedad que tengan. Luego las contrastará con nuestras respuestas.

\section{Frases para analizar}

1. ANA: "La educación en valores no debe darse en la escuela sino en casa o en el tiempo libre. La escuela está para otras cosas. Mi alumnado encontrará trabajo de contable si sabe calcular el IVA no si es solidario".

2. BEA: "Los valores que se dan en el cole no funcionan. Si un niño me quita el juguete y no me defiendo me quedo sin él, o si un país me invade y recurro a las buenas palabras no evitaré que me invada. Es absurdo hablar de dar el 0,7\% de ayuda al desarrollo si el sistema económico capitalista prima al país o empresa que explota más a los demás".

3. CARLOS: "En nuestro proyecto educativo y curricular de centro señala que "es una de las prioridades desarrollar una cultura de la paz y la solidaridad" por ello yo en clase les explico problemas como el hambre en países del Sur o el racismo en España".

4. DIEGO: "Eso de educar en valores es una trampa porque ¿En qué valores educo? ¿Un profesor marxista educa en el marxismo y uno católico en el catolicismo? Esa es una decisión de la familia o como mucho de centros que optan por ser confesionales".

1. Para contrastar alguna de las ideas expuestas en el artículo se puede recurrir a: PANIEGO, José Ángel (1999), Como podemos Educar en Valores. Madrid: CCS. En él se puede encontrar una discusión ampliada de los aspectos más conflictivos así como actividades didácticas para trabajar los valores. La primera aproximación a los resultados de este modelo educativo global puede encontrarse en PANIEGO, J. A., SEVILLA, B. y BOLLAÍN, S. (1998) Educapaz. Una formación integral de actitudes para el cambio social. En SÁNCHEZ TORRADO, Santiago. Ciudadanía sin fronteras. Cómo pensar y aplicar una educación en valores. Bilbao: Desclée De Brouwer. También recomendamos al lector que quiera profundizar en las técnicas clásicas de Educación en Valores acudir a: MARTíNEZ, Miquel y PUIG, Josep M. (coor) (1991), La Educación Moral. Perspectivas de futuro y técnicas de trabajo. Barcelona ICE-Grao. Y también a BUXARRAIS, Mำ Rosa (1997), La formación del profesorado en educación en valores. Propuesta y materiales. Bilbao: Desclée De Brouwer. 
5. ELENA: "Yo les mando trabajar en equipo y no funcionan, hago debates y me encuentro con frases como "los negros a su país". Creo que no funcionan estos métodos modernos".

6. FELIX: “¡Dejémonos de zarandajas! Hay que elevar el nivel de contenidos que ya bastante bajo está y no andar con tanta tontería de los valores".

Reflexione el lector sobre estas frases antes de pasar a leer la respuesta que damos a las opiniones de cada uno de los personajes. Durante este diálogo iremos configurando nuestra propuesta de Educar en Valores.

1. ANA. El problema que nos plantea Ana nos lleva a la pregunta ¿Para qué educamos?

Si educamos a las personas es para prepararlas para el día de mañana. Por ello debemos desarrollar:

a) Un equilibrio personal. Si somos infelices y estamos desesperados algo falla en nosotros, no hemos sido preparados para la vida adulta.

b) Un correcto desempeño social. Si no nos socializamos adecuadamente en los círculos cercanos (amigos, familiares) o lejanos (como ciudadanos que participan en la gestión de su planeta) algo falla.

c) Un correcto desempeño laboral. Sobre este último señalemos que no es sinónimo de conocimientos. Existe un cierto divorcio entre rendimiento académico y profesional. La pista para comprender este divorcio puede dárnosla la observación de cierto responsable de una empresa informática: "Si un trabajador no conoce un programa que usa la empresa le damos un curso y en una semana aprende a manejarlo. Pero si no sabe trabajar en equipo, yo no le puedo enseñar".

Como vemos, educar es educar no solo contenidos sino también actitudes y conductas. ¿Quién debe encargarse de esa formación? La escuela no es el actor único. Está la familia, hay asociaciones que trabajan en el tiempo libre... Los diversos agentes educativos podemos y debemos coordinarnos. Por ejemplo, con las familias puede haber un flujo de información y asesoramiento. Existen programas como "Preescolar na casa" ${ }^{2}$ que se desarrolló en Galicia, promovido por la Consellería de Educación, donde era la familia la que trabajaba los contenidos de educación infantil, asesorada periódicamente por maestros que acudían a visitarla.

Hay que coordinarse sí, pero no evadir responsabilidades. Si educar es prepar a las personas para la vida, toda formación que no cumpla este objetivo es simplemente una mala formación. Debemos replantear los currícula educativos eliminando los elementos de menor utilidad y dando paso a elementos más útiles, entre los que vimos se deben encuentrar valores traducidos a actitudes y conductas. Para ello, el profesorado debe sentirse un formador integral de personas no un reproductor de contenidos de disciplinas decididos hace siglos. Sin este cambio, tendremos una escuela que no prepara para la vida y por tanto una escuela de mala calidad.

2. Puede verse en: PAZ COMBARRO, M. a Sara y ARMESTO RODRÍGUEZ, Celia (2000). Preescolar na Casa. Educación Infantil familiar; una utopía realizable. Madrid: Cáritas Española. O también a través de Internet en: www.lugo-com.com/us/preescolar.na.casa 
2. BEA. Bea nos plantea varios problemas. El primero es el de la validez de los valores impartidos en el aula, que muchas veces no son validos para la vida. Si un niño me quita el juguete y me lo dejo quitar soy un niño pasivo, soy ese niño del cual se burlan los niños. Nadie pensará que esta situación es buena. Por si acaso comentaré que es una situación que correlaciona con los mayores problemas psicológicos y con el mayor fracaso escolar. Cuando un profesor se limita a decir: "niño no se pega" está construyendo niños pasivos. Está invitando, o a crear niños inadaptados y fracasados que usan estrategias inválidas; o al cinismo, al ver que lo que le dicen en la escuela no es válido para la vida. Y si esto no es válido, ¿por qué va a creer otras cosas que se digan en el aula? Posiblemente estemos contribuyendo a crear un rechazo generalizado de la institución escolar y del aprendizaje en general. "Afortunadamente" ahí suele estar el padre que dice "tu pega antes de que te peguen". Se supera así la pasividad, pero... si yo pego el otro puede que me pegue, o puede que rompamos el juguete por el que luchamos, o puede que un adulto me castigue. En general, se ha observado que el chico que pega no es ni el más querido, ni el que es más capaz de desenvolverse en la vida, ni el líder y por supuesto tiene alto fracaso escolar. ¿Cómo es el chico exitoso en estos campos? Por ejemplo, ¿Qué haría este chico en la situación descrita en que otro le quita el juguete? Piense el lector cómo afrontar esta situación.

Diversos estudios muestran ${ }^{3}$ que los chicos queridos y exitosos son aquellos que:

- Intercambian (por ejemplo, dicen: "este juguete es más chuli que el que me has quitado, lo has visto"...y cuando ha captado la atención del otro dice: "bueno, te lo cambio"; pero no dicen: "pues el día que traiga algo ya veras").

- Juegan juntos (por ejemplo, dicen: "me has cogido la pelota pero ¿A que no me metes gol?"; pero no dicen: "anda me dejas jugar").

- Y por supuesto piden las cosas sin enfadarse pero con firmeza (dicen: "eso lo tenía yo" pero no dicen: "porfa... devuélvemelo").

En resumen, son personas asertivas que cooperaran porque saben conseguir que se coopere; porque tienen habilidades sociales exitosas validas para la vida fuera del aula.

Y en el caso de un país invadido; ¿Que hacemos? ¿Dejarnos invadir no parece lo óptimo? Defendernos militarmente por el contrario equivale a muerte y destrucción, y no garantiza la victoria del más justo ¿Qué otra cosa podríamos hacer? ¿Se le ocurre algo al lector?

Podemos darnos cuenta de que: si un tirano (invasor extranjero o nacional) puede mandar es porque se le obedece. Puede ocupar el ministerio de industria, pero si los trabajadores hacen huelgas no obtendrá los bienes que busca; puede pedir impuestos,

3. Para ver el tipo de interacción exitosa y la importancia de las mismas podemos ver los trabajos de Díaz Aguado: DíAZ-AGUADO, María José (1986), El papel de la Interacción entre iguales en la adaptación escolar y el desarrollo social. Madrid: C.I.D.E. y DÍAZ-AGUADO, María José (1988) La interacción entre compañeros: Un modelo de intervención educativa. Madrid: C.I.D.E. También es muy interesante el artículo HARTUP, Willard W. (1978) Children and their friends en McGURK, H. (Ed.), Issues in chilhood social development. Londres: Methuen. Que nos habla de la importancia de esta adecuada socialización infantil. 
pero si nadie los paga no puede cobrarlos; puede imponer sus productos, pero si todo el mundo los boicotea no podrá obtener beneficios; y por supuesto puede intentar tomar represalias contra los cabecillas, pero si todos desobedecen no puede meterlos en la cárcel. Esto que se denomina técnicas noviolentas, y que suena a "sueños utópicos" no es algo utópico (etimológicamente algo sin lugar) sino real que ya ha tenido sus lugares y tiempos. Cuando Gandhi expulsa a los ingleses de la India es esto lo que hace, cuando en El Salvador cayó el dictador Martínez en 1944 o en Guatemala Jorge Ubico son estos medios los usados por la población. No entraremos ahora a valorar en qué situaciones es aplicable la no violencia y en cuales no. Señalar, eso sí, que no es un tipo de acción solo valida con un invasor civilizado, como los ingleses en la India. Ni los ingleses eran tan caballerosos (llegaron a cercar una manifestación en una plaza y disparar sus ametralladoras produciendo miles de muertos y heridos); ni tiranos como Adolfo Hitler pese a su falta de escrúpulos dejaron de sufrir los efectos de resistencias noviolentas, como las de Dinamarca o Noruega. Hay incluso testimonios de responsables nazis diciendo que preferían enfrentarse a resistencias violentas. Lamentaremos aquí el desconocimiento de la noviolencia incluso entre el profesorado de historia. Este dedica horas a explicar guerras que suponen un mero redondeo de fronteras que sólo dura hasta que en la siguiente guerra los vencidos, ya repuestos, dejaban las cosas como al principio. Sin embargo, olvida mencionar que hechos importantes (desde la creación del derecho romano hasta los derechos de minorías negra o hispana en EEUU), son fruto de acciones no violentas. Igual que en nuestros periódicos sienten "fascinación por la violencia", y así la partición de Yugoslavia ha ocupado muchas páginas mientras que la de Checoslovaquia apenas unas líneas; en nuestros libros de historia se dedicarán páginas a la independencia italiana de Austria (algo sangriento), y muy poco a la autonomía primero e independencia después de Hungría (algo similar pero pacífico). ${ }^{4}$

En resumen, ni en las situaciones cercanas, ni al analizar los hechos lejanos, mostramos estrategias óptimas. En lo cercano solicitamos pasividad y no enseñamos una postura asertiva-cooperativa. En lo lejano por el contrario hacemos apología de lo violento y no mostramos la postura noviolenta. El alumno un poco espabilado, hará la lectura: "me dices que sea pasivo, pero me muestras que lo que funciona es la violencia". ¡Nosotros mismos estamos descalificándonos! Si queremos generar conductas válidas fuera del aula, debemos dotar al alumnado de habilidades para no ser pasivo ni agresivo sino asertivo-cooperativo y mostrar en diversos niveles, cómo esas estrategias funcionan.

Contrastemos en más detalle la cooperación y la competición. En el modelo competitivo la persona o grupo intenta imponer sus deseos a los demás por la fuerza. Así, el país rico se impone al pobre (problema del desarrollo), la raza, ideología o religión dominante a las otras (racismo, teocracia...) o el sexo con más poder, el masculino usualmente, margina al otro (problema del sexismo). Esto genera tensiones que se resuelven por medio de la violencia militar o policial (problema de la paz). Esta vio-

4. El lector puede encontrar una información algo más detallada sobre la noviolencia en los trabajos del Colectivo Noviolencia y Educación especialmente en CNE (1994), Alternativas al militarismo. Madrid: Colectivo Noviolencia y Educación. C/ Padre Piquer 54, 2oD. 
lencia se ensaña sobre todo con los débiles, pero también tiene otros efectos. La tensión por acaparar recursos hace que estos se destruyan o no se generen eficientemente, y que el entorno no se conserve (problema ecológico). Los privilegiados también sufren de vuelta el efecto de la competición: el sexismo hace que muchos varones no sepan freír un huevo y tengan que pagar un restaurante; la degradación de recursos hace que el aire que respira sea más sucio; el problema del desarrollo hace que el Sur no pueda pagar la deuda o comprar productos del Norte, aumentando el paro y la crisis económica de los ricos; la violencia reactiva de los oprimidos contra los opresores puede llegar a salpicar al poderoso; y sobre todo, la dinámica de ver al otro como adversario produce tensiones y limita el desarrollo personal.

Este modelo social no sólo es insatisfactorio para todos, sino que diversos analistas señalan que es insostenible. La tierra no soporta la tensión de un modelo donde se esquilman sistemáticamente sus recursos y donde se generan toneladas de residuos nocivos. $^{5}$

Frente a este modelo, está la opción cooperativa. En ella se busca la igualdad entre todos (países, personas, razas, sexos...) de forma sostenible. Para ello, busca producir eficientemente y distribuir adecuadamente los recursos entre quienes los necesiten. Incluso cuando se enfrenta a la competición, su objetivo no será meramente vencer. Si se ve impelido a usar la fuerza, esta utilizará el mínimo de violencia y con el objetivo de conseguir una situación de igualdad, donde se restablezca el diálogo para lograr una situación de más justicia para todos. ${ }^{6}$

Dos conclusiones surgen de este análisis:

a) Educar en valores no es educar para "hacer caridad", sino para darnos cuenta de que nos interesa hacer un mundo más justo. Al país rico le afecta la pobreza del Sur, al varón le afecta el sexismo o a todos la degradación ecológica. Si nos ocupamos de problemas sociales es interés de todos, pues todos vamos en un mismo barco, llamado planeta tierra. La caridad es algo voluntario, un añadido ético que podemos conceder o no. La necesidad de cambiar el modelo por interés común, convierte la reflexión y la acción ética en una preocupación personal ineludible y la educación en valores en necesidad formativa central.

b) Todos los temas que componen la educación en valores están interrelacionados. Educar en valores adecuadamente pasa por generar una actitud global de cooperación no meramente por un trabajo parcial de alguno de los temas. Puede y debe haber momentos específicos para trabajar cada tema y hay especialistas y publicaciones especializadas. Por poner un ejemplo, muchas ONG's están elaborando materiales de Educación para el Desarrollo. Se trata de materiales especializados y que como tal deben ser una parte del contenido de valores y no el todo. No obstante, desde INTERMÓN y desde muchas otras ONG's, cada vez

5. Análisis que muestran la imposibilidad de mantener el modelo socioeconómico actual son innumerables. Quizás se puede resaltar: MEADOWS, Denis et al. (1972), Los límites del crecimiento. México: Fondo de Cultura económica. y MEADOWS, Donella et al. (1992), Mas allá de los límites del crecimiento. Madrid: El País-Aguilar.

6. Para un análisis estratégico de la cooperación frente a la competición podemos ver: AXELROD, R. (1986) La evolución de la cooperación. Madrid: Alianza. 
se es más consciente de la necesidad de materiales que tengan en cuenta dimensiones interrelacionadas. Así, se van introduciendo análisis de género, análisis sobre la violencia militarista, sobre degradación ecológica y muchos otros. Todavía la mayoría de las ONG's distan bastante de una concepción global del fenómeno educativo de los valores; pero, las que quieran tener incidencia en el mundo formativo deben acercarse más a esta idea.

Volviendo a nuestra BEA, su última frase afirmaba que es absurdo dar el $0,7 \%$ si estamos en un sistema que explota a las personas. Efectivamente, si nuestro sistema se basa en la competición (y muchos ideólogos del capitalismo neoliberaral señalan que el sistema necesita la desigualdad para funcionar) es absurdo hablar de cooperación. Pero el problema no es que la cooperación no funcione y el modelo sí. El problema es que estos modelos están mostrando no funcionar y generar desigualdades e injusticias. Dado que estos sistemas no funcionan Educar en Valores es educar personas que se comprometan con un cambio de la sociedad. Es decir, fomentar una autentica ciudadanía democrática, que no es la que se limita a votar cada cuatro años, sino la que día a día se preocupa por su sociedad y actúa para mejorarla. Muchas veces se ha hecho una Educación en Valores "light". Una educación institucionalizada que pretende evitar críticas al sistema. Así, Educar para la Paz la convierten en educar para que los niños no se peguen, pero no para que se critique el gasto militar; o Educar para el Desarrollo la convierten en educar para hacer caridad con el Sur, no para cambiar la estructura de consumo del Norte responsable del empobrecimiento del Sur. Esta educación "light" esta abocada al fracaso. Si existe incoherencia entre las dimensiones de lo cercano y lo lejano no existirá aprendizaje. Si les imbuimos que la violencia funciona en lo lejano ¿Cómo esperamos que no la aplique a lo cercano? Si reprimimos las criticas al modelo social, al escolar o a la actuación del profesorado ¿Esperamos que critique las actuaciones injustas del niño de al lado? ¿O que sea crítico con el que le ofrece droga? Si además damos unos valores que son inválidos para la vida ¿Esperamos de la persona algo distinto de la incredulidad?

No sólo es una necesidad formativa de las personas. La sociedad necesita personas críticas, la escuela necesita personas críticas. Si nadie hubiera criticado cosas y propuesto cosas distintas seguiríamos en la cultura del paleolítico. Una sociedad cerrada sobre sí misma, una sociedad sin crítica y evolución, está condenada a la extinción. Más adelante veremos que una de las necesidades es aprender a desobedecer y criticar, no como una forma de enfado y violencia, sino como una forma de aportar ideas y acciones que mejoren lo que esta mal.

3. CARLOS. Carlos plantea en primer lugar la importancia de recoger en los diseños educativos la Educación en Valores. Esta no puede funcionar si no está presente en todo el quehacer educativo y de forma convenientemente organizada y programada. No obstante, fijémonos en cómo se menciona: "cultura de la Paz y la Solidaridad". Nos preguntamos ¿Qué es paz? ¿Es crear más ejércitos y armas nucleares que defienden la paz o es hacerse insumiso para abolir los ejércitos? ¿Qué es solidaridad? ¿Es boicotear a multinacionales que explotan mano de obra infantil o es dar ropa que nos sobra a una ONG? Y sobre todo: ¿Tenemos todos claro qué actividades realizar para educar en estos valores? Como vemos Educar en Valores no es educar en unas palabras abstractas sino en valores que 
se operativizan en actitudes y conductas concretas y con un conjunto de prácticas didácticas definidas.

Otro aspecto que señala Carlos es que él "explica". En general, no basta explicar y saber cosas. En los años 70 se encontró en los Países Nórdicos y Gran Bretaña, que ciertos niños podían saber perfectamente la diferencia de renta de los países del Sur, la gran cantidad de enfermedades asociadas a la pobreza y mucha otra información; pero eso, no bastaba para hacerles concienciarse y actuar contra ese problema. Si queremos formar actitudes y conductas no basta dar información sino que es preciso tener en cuenta los tres aspectos asociados a la actitud: cognitivo (saber cosas) afectivo (sentir vivenciar cosas) y conductual (hacer cosas).

Esto implica un replanteamiento general del quehacer educativo. No se puede hablar de cooperación internacional si nuestros métodos no implican la cooperación con el vecino de pupitre; no se puede hablar de participación si nuestra clase no es participativa; o no se puede dar una imagen positiva del Sur si en nuestros textos el Sur solo sale como paradigma de atraso o de exotismo. Por ello, hay que cambiar:

a) La metodología del aula que debe ser activa, participativa, cooperativa y constructivista, dando el protagonismo al alumno. Por ejemplo usando programas de aprendizaje cooperativo o tutorías entre iguales. (Supongo que el lector entenderá ahora la forma dada a este artículo, que intenta ser coherente con esta metodología dándole cierta participación al lector).

b) La organización escolar que debe ser más democrática para formar una comunidad justa ("just comunity").

c) La evaluación que debe orientarse a medir la capacidad real del alumno y no sólo la memorización de contenidos durante el día del examen.

d) Analizando el currículum oculto y descubriendo los valores que transmiten nuestros textos, nuestras actitudes y nuestro quehacer educativo.?

4. DIEGO. Diego plantea el problema de la neutralidad frente al adoctrinamiento. En primer lugar démonos cuenta que su opción por el neutralismo es falsa. Por

7. Los análisis sobre los diferentes aspectos mencionados son innumerables. Entre las principales recomendaciones a parte del mencionado PANIEGO, José Ángel (1999), O.C. pp 193-251. Para análisis de los programas de Aprendizaje cooperativo: WEBB, Noreen M. (1984), Interacción entre estudiantes y aprendizaje en grupos pequeños. Infancia y Aprendizaje, no 27-28, pp.159-183. Para análisis de las tutorías entre compañeros podemos consultar: VARGAS CANO, J.D. y VILLARES BERMEJO, L. (1999), Funciones y estrategias de la guía-tutela entre iguales en las primeras edades. Contextos Educativos, no 2, pp. 93-105. Para la idea de comunidad justa podemos acceder a WASSERMAN, E. R. (1977), The development of an alternative high school based on Kolberg's just community approach to education. Boston: University School of Education. Sobre un análisis de la organización escolar y el diseño para su adaptación a la educación en valores podemos ver: YUS, Rafael (1996), Temas transversales: Hacia una nueva escuela. Barcelona: Graó. Sobre el curriculum oculto existen a su vez muchos análisis más que globales sobre diferentes temas por citar solo dos ejemplos la presencia de la educación intercultural y para el desarrollo en los libros de texto podemos leer: CRUZ ROJA (1990), Tercer Mundo y Racismo en los libros de texto. Madrid Cruz Roja. Y sobre sexismo podemos leer: MORENO, M. (1986), Cómo se enseña a ser niña: el sexismo en la escuela. Barcelona: Icaria Finalmente sobre técnicas evaluativas podemos acceder a: BOLIBAR, Antonio (1995), La evaluación de valores y actitudes. Madrid: Anaya. 
ejemplo, no educar para la igualdad entre los sexos y dejar que la niña vea en casa la reproducción de roles sexistas, no es ser neutral, es caer en el reaccionarismo; o no educar para el desarrollo pero dejar que las imágenes que ve en las películas de las gentes del Sur, sean las de seres atrasados e ignorantes hasta rayar la estupidez, no es ser neutral; no hablar de interculturalidad pero dejar que oiga a su familia que los gitanos y los negros roban y traen droga, no es ser neutral. No trabajar los valores en general, no es ser neutral, es dejar que perviva el más rancio conservadurismo.

Por otro lado, no podemos dejar de transmitir valoraciones. Si hacemos trabajo en equipo enseñamos a cooperar; si dos alumnos tienen un conflicto, según cómo actuemos ante él, estaremos ejemplificando la forma de actuar ante los conflictos; o si nuestro lenguaje es sexista estaremos transmitiendo el sexismo. Es pues falsa la idea de neutralidad absoluta pues toda educación es educación en valores.

Entonces ¿qué hacer? ¿Dejar la decisión en manos de la familia? ¿Ofrecer opciones ideológicas-educativas a la carta, en aras de un pluralismo? En primer lugar ¿Qué pasa si una familia quiere una educación sexista o racista? No es una cuestión de meramente delegar en la familia. En segundo lugar, es común confundir pluralismo con pluralidad de monolitismos. Tener muchas opciones, pero cada una de ellas cerrada sobre sus dogmas, es una pluralidad de monolitismos. Obtendremos personas dogmáticas, con dogmas distintos eso sí, pero no personas abiertas. Una educación plural es la que ofrece todos los puntos de vista no al hijo de marxistas el marxismo y al hijo de anarquistas el anarquismo sino que ambos vean el marxismo, el anarquismo, el neocapitalismo y todas las opciones posibles. O tampoco es plural que el hijo de los budistas conozca sólo el budismo y el hijo de ateos el ateísmo. El pluralismo es que todos conozcan el ateísmo, el budismo, el cristianismo, el agnosticismo y todas las opciones religiosas. Como vemos el primer paso para lograr una educación no adoctrinadora es una educación que lleve a conocer todos los puntos de vista.

Pero va más allá, no es sólo que haya oído todas las opciones. Es una educación que ponga a la persona en disposición de optar por actitudes y conductas más maduros. Esto, nos lleva a algo más que a meramente exponer todos los puntos de vista. Pasa por tener una acción pedagógica con tres pasos:

a) Vivenciar/problematizar. Vivenciar implica conocer de un modo activo que incluya la dimensión afectiva. Igual que una persona que no se ha enamorado no sabe lo que es el amor, aunque leyese todas las teorías psicológicas sobre el tema; no podremos decir que alguien comprende un hecho social si no se impregna de él y comprende las dimensiones emocionales del mismo. Problematizar por su parte es mostrar problemas, cosas que no funcionan en nuestra forma de ser y actuar. Por ejemplo, si alguien afirma que su familia no es sexista, pero se da cuenta de que en su casa las tareas domésticas las hacen las mujeres, encontrará que hay algo que no funciona, una incoherencia; y necesitará reelaborar sus actitudes y conductas a la luz del nuevo dato descubierto.

Dinámicas de rol, audivisuales, textos con fuerte carga emocional pueden ser elementos útiles para realizar este trabajo. 
b) Reflexionar. Evidentemente, debe realizarse un proceso de análisis personal. Este proceso debe implicar tanto la búsqueda de información (de toda la información), como el contraste con las ideas de los demás.

c) Desarrollar y entrenar conductas adecuadas. Este último paso, frecuentemente olvidado, es fundamental. No basta decir, bueno ya sabemos lo que pasa. Es necesario, poner en practica nuevas actitudes y conductas. Por ejemplo, si hemos visto el problema del desarrollo podemos poner en práctica muchas acciones que pueden ir desde apoyar a asociaciones como ONG's, hasta tener un consumo responsable, o escribir protestando por la política de determinadas empresas o administraciones. Y muchas de las conductas deben entrenarse. Por ejemplo, aunque decidamos no actuar con violencia, hasta que nos habituemos a una reacción no violenta pasará un tiempo en que debemos entrenar las conductas adecuadas. Así, desde INTERMÓN, los materiales didácticos proponen acciones que el alumnado puede realizar (escribir cartas, realizar pancartas, acudir a parlamentos para hacer sugerencias a nuestros legisladores...).

Para generar personas que no hayan sido adoctrinadas y que tengan unas actitudes y conductas construidas personalmente, más maduras y solidarias es necesaria una educación que haga vivenciar/problematizar, lleve a una reflexión profunda y dé paso a conductas que mejoren la realidad social.

No obstante, nos preguntaremos en la siguiente frase, si todavía algo falta.

5. ELENA. Elena nos muestra que a veces, no funciona todo lo bien que sería de desear, un modelo como el propuesto. Posiblemente, a Elena simplemente le falte experiencia para poner en práctica estos sistemas, pero probablemente no se trate de eso sólo, sino que olvidó trabajar algún elemento necesario para que funcione su metodología. Elena debe tener en cuenta las dimensiones:

a) Realidades sociales. Señalamos la necesidad de trabajar una serie de realidades sociales interrelacionadas (Desarrollo, ecología, paz...).

b) Medios de socialización. Decíamos que las películas que ve, los valores de la familia, los juegos a los que juegue... transmiten valores. Con frecuencia, valores mucho más firmes que los transmitidos en el centro educativo. Si queremos trabajar el nivel de las realidades sociales, debemos comprender que se asientan en las ideas imbuidas por los diferentes medios de socialización y así:

- Enseñarle a ser crítico con los valores que recibe para ser capaz de defenderse de influencias negativas.

- Seleccionar lo positivo de los medios de socialización para acercarlo al aula.

- Coordinarse los agentes socializadores (especialmente familia y escuela) de modo que haya concordancia y asesoramiento para la acción formativa.

c) Habilidades instrumentales. Si nos fijamos hemos hablado de analizar de forma crítica los valores de los medios de socialización. Este análisis para que de verdad sea crítico y desprejuiciado precisa el haber trabajado una serie de habilidades instrumentales: 
- La capacidad de ser crítico y desobediente de forma constructiva. Tradicionalmente o no actuamos contra la injusticia o nuestra reacción es una mera canalización de la rabia. Normalmente la persona que decimos es muy obediente es la que es incapaz de crítica (pasiva) y la que decimos que es muy desobediente es la que no ha aprendido a ser constructiva (es violenta). Frente a ellas es preciso una persona que aporte ideas constructivas y canalice la desobediencia o la crítica a la consecución de una situación de más justicia.

- La capacidad de regular los conflictos. El conflicto se ha comprobado que es fundamental para la maduración personal. Regular adecuadamente nuestros conflictos nos llevará a evolucionar como personas y a aprender a enfrentarnos a situaciones conflictivas en otros niveles.

- La capacidad de tomar decisiones consensuadas. La construcción de actitudes se ha comprobado es mucho mayor en un grupo de iguales que interactúa. Pero para que esa actuación sea positiva, precisa aprender a tomar las decisiones, de modo que se alcancen consensos, válidos para todos y no sólo para el líder o para un grupo (incluso aunque ese grupo sea el mayoritario que ha ganado una votación).

- La capacidad de superar los prejuicios. Este es un elemento central. De niños aprendemos una forma de ver el mundo en la llamada socialización primaria. A partir de ahí, todo lo veremos con esos prismas adquiridos. Recuerdo un chiste en que un cura católico entraba en un prostíbulo. Una persona atea que lo ve entrar piensa "¡Hay que ver cómo son estos curas! Mucho predicar moral pero mira". Otra católica por su parte piensa: “¡Hay que ver cómo son estos curas! Hasta a estos sitios vienen a ejercer su sacerdocio". Nosotros señalaremos ¡Hay que ver cómo son estas ideologías! Que hasta los hechos puros los reinterpretan para que sean acordes a sus postulados. Si queremos una persona que evalúe la realidad de forma abierta y desprejuiciada, uno de los elementos centrales será el trabajo de los prejuicios. Debemos mostrar la existencia de otras perspectivas, válidas y distintas de la nuestra, como forma de acostumbrarnos a evaluar todas las perspectivas antes de juzgar o actuar.

d) Actitudes cooperativas grupales. Los niveles anteriores, precisan que en el grupo de iguales se dé una actitud coherente. Si con los cercanos sabemos cooperar, o tenemos un trato no racista y sexista, veremos una coherencia con lo que se quiere desarrollar a otros niveles. Así mismo tendremos un grupo de referencia, abierto a la construcción de nuevas ideas y valores. Por ello, es preciso desarrollar: el conocimiento, la comunicación, la confianza, el contacto y la estima y la capacidad de cooperar.

e) Desarrollo personal. Y finalmente, todo se asienta sobre unas determinadas capacidades personales. Por ejemplo, si tenemos baja autoestima es posible que la aumentemos haciéndonos racistas para sentirnos "superiores a negros, moros, mujeres...". Cualquier intento de eliminar la actitud racista, debe tener en cuenta que ésta es útil para elevar mi autoestima, y que me resistiré a cambiar una actitud aparentemente tan útil, a menos claro está, que me 
enseñen otro modo de elevar mi autoestima. O por ejemplo, si no tengo un buen control emocional antes de analizar las cosas en profundidad me habré dejado llevar por mis emociones. Con ello, se impide una reflexión madura que me permita acercarme a nuevas actitudes y conductas. Un intento de seleccionar las variables personales que más incidencia tienen en la Educación en Valores, nos ha llevado a comprobar la importancia de trabajar: El autoconocimieto, la autoafirmación y autoestima, el autocontrol emocional, el autocuidado, la empatía (incluyendo la capacidad de reconocer y transmitir emociones) y el desarrollo del razonamiento moral y la creatividad. ${ }^{8}$

Solo un desarrollo armónico de todas estas dimensiones hará que las personas desarrollen actitudes y conductas solidarias.

6. FELIX. Finalmente el último texto nos plantea la dicotomía que sienten muchos profesores entre educar en el curriculum tradicional o "perder el tiempo" haciendo Educación en Valores. Más, en un momento en el que perciben muchos docentes, que ha caído el nivel académico. Si bien ya dijimos al principio, que la educación debe ser algo más que formar contenidos incluso para el éxito laboral, señalemos varias cosas:

a) En primer lugar, cierta caída en el nivel académico es falsa. Por ejemplo, con la ampliación de la edad de escolarización muchos profesores se encuentran con alumnado con bajo nivel. No es que antes estuvieran mejor formados, sino simplemente que antes hubieran sido expulsados del sistema educativo y el profesor no los vería. Además, parte de ese descenso es aparente, quizás no hayan memorizado tantas cosas pero sepan procesar y organizar mejor la información. Dicho de otro modo y si se me permite la alegoría: hoy en día preferimos que el alumno, en vez de saber de memoria cuatro páginas de la guía telefónica, sepa buscar un teléfono en la misma. Quizás por eso sea ahora más raro el alumno que en secundaria era brillante (cuando tenía que memorizar el texto del examen) pero en la Universidad (enfrentado a otra forma de estudiar) o en el trabajo (donde no se prima el memorismo) fracase estrepitosamente.

b) En la caída académica existen factores extra-académicos. La violencia social es mayor no sólo dentro de la escuela, la valoración de la escuela es bastan-

8. Seleccionar entre los diversos estudios que han hablado de las diversas dimensiones expuestas es arduo. De nuevo un acercamiento global lo encontraremos en el libro ya citado PANIEGO, José Ángel (1999) O.C. Un popular estudio sobre la importancia de las dimensiones personales no sólo en la escuela esta en GOLEMAN, Daniel (1996), Inteligencia Emocional. Barcelona: Kairós. Sobre conflicto podemos consultar: TORREGO, Juan Carlos (coor.) (2000), Mediación de conflictos en instituciones educativas. Madrid: Narcea. Sobre desobediencia: BETZ, G. (1981), Educación para la desobediencia. Ediza. Herria 2000. Sobre prejuicio un acercamiento aunque no didáctico podemos encontrarlo en ALLPORT, G. W. (1971), La naturaleza del prejuicio. Buenos Aires: Eudeba. Para las grandes dimensiones sociales podemos señalar: sobre educación para el Desarrollo: MARHUENDA, Fernando. La educación para el desarrollo en la escuela. Posibilidades e interrogantes. Barcelona: INTERMÓN. Y sobre Educación para la Paz S.E.P.D de A.P.D.H. (1994), Educar para la paz. Una propuesta posible. Madrid. Los libros de la catarata. 
te negativa; ya no se ve tan claramente, como forma de promoción social, el estudio.

c) Pero finalmente ¿es la Educación en Valores la causante de este malestar?. No, salvo que la identifiquemos con una educación en que guardamos "ratitos" para hablar de "cosas que se salen del programa". Un problema de los modelos que han fracasado, ha sido que han entendido la educación en valores como algo que sólo se toca en algún momento (Por ejemplo tutorías, alguna sesión suelta, alguna jornada cultural...) y centrándose sólo en algún aspecto (un profesor trabaja por ejemplo sexismo y otro autoestima sin coordinarse y olvidando una formación en valores más global). No ver la educación en valores de forma integral, es una formación deficitaria, igual que sería la pretensión de enseñar física olvidando las bases matemáticas o los campos comunes con la química, una invitación al fracaso educativo.

Entendemos la Educación en Valores como:

a) Un replanteamiento de toda la práctica educativa: Modelos de aprendizaje, modelos de organización escolar, evaluación o curriculum oculto.

b) Un trabajo de construcción personal que se produce en grupo trabajando los tres pasos: Vivenciación/problematización, reflexión, acción.

c) Un trabajo equilibrado de todas las dimensiones: personal, grupal, habilidades instrumentales, medios de socialización y grandes realidades sociales.

d) Una educación entendida como modelo para preparar a las personas para la vida futura incluyendo involucrarse en procesos de cambio social.

La práctica de la Educación en Valores, como la hemos definido, genera:

a) Personas con valores más solidarios y tolerantes, asumidos y construidos no meramente indoctrinados.

b) Una mejor preparación para el desempeño futuro personal, social o laboral.

c) A corto plazo una mejora en el clima escolar y en la motivación que se traduce en mejor rendimiento escolar. ${ }^{9}$

Como vemos un buen trabajo en educación en valores lejos de ser un añadido más es fundamental para la persona madura, la escuela de calidad o la sociedad en progreso.

9. Son múltiples las propuestas que desarrollan alguna de las partes aquí propuestas con resultados satisfactorios. Nos limitaremos a mencionar dos programas que quizás sean los más fértiles en implantación y en generar investigación. Los trabajos de Kolberg y sus seguidores sobre el razonamiento moral. Por ejemplo en HERSH, Richard H. et al. (1988), El crecimiento moral. De Piaget a Kolberg. Madrid: Narcea y también en DÍAZ-AGUADO, María José (1995), Educación y razonamiento moral. Deusto: Ediciones Mensajero. Por otro lado y en una línea bien distinta el programa de filosofía para niños de Lipman. El programa para los diversos niveles (tanto guías de profesor como cuentos para el alumnado)se encuentra en Ediciones de la Torre. Una síntesis del pensamiento de su autor esta en LIPMAN, Matthew (1997) Pensamiento complejo y educación. Madrid: Ediciones de la Torre. 